\title{
MR-plastination-arthrography: a new technique used to study the distal tibiofibular syndesmosis
}

\author{
John J. Hermans • Noortje Wentink • \\ Gert-Jan Kleinrensink • Annechien Beumer
}

Received: 14 October 2008 /Revised: 23 November 2008 / Accepted: 3 December 2008 / Published online: 9 January 2009

(C) The Author(s) 2008. This article is published with open access at Springerlink.com

\begin{abstract}
Purpose The purpose of this study was to describe a new technique called MR plastination arthrography to study both intra- and extra-articular anatomy.

Materials and methods In six human cadaveric lower legs MR arthrography was performed in either a one-step or two-step procedure. In the former a mixture of diluted Gadolinium and dyed polymer was injected. In the latter the dyed polymer was injected after arthrography wih diluted Gadolinium. Three-millimeter slices of these legs, obtained in a plane identical to that of the MR images, were plastinated according to the E12 technique of von Hagens. The plastination slices were subsequently compared with the MR images. Results The one-step procedure resulted in an inhomogeneous arthrogram. The two-step procedure resulted in a good correlation between the high-resolution MR images and plastination slices, as expressed by a good comparison of anatomic detail of the small syndesmotic recess.

Conclusions Images of the distal tibiofibular syndesmosis obtained with plastination arthrography correlated well with images acquired by MR arthrography when performed in a two-step procedure.
\end{abstract}

J. J. Hermans $(\triangle) \cdot$ N. Wentink

Department of Radiology,

Erasmus University Medical Center,

's Gravendijkwal 230, P.O. Box 2040, 3000 CA Rotterdam,

3015 GD Rotterdam, The Netherlands

e-mail: j.j.hermans@erasmusmc.nl

\section{G.-J. Kleinrensink}

Department of Neurosciences,

Erasmus University Medical Center,

Rotterdam, The Netherlands

\section{A. Beumer}

Department of Orthopedics, Erasmus University Medical Center, Rotterdam, The Netherlands
Keywords Morphology · Plastination · MRI · Arthrography $\cdot$ Tibiofibular syndesmosis

\section{Introduction}

MR arthrography is an accepted technique to study intraarticular pathology. In patients with an injury of the distal tibiofibular syndesmosis the joint space and its recesses may be involved. Therefore the normal anatomy not only of the syndesmotic ligaments but also of the joint space and of the height and contour of the syndesmotic recess is of interest. One way to study the anatomy is by using the technique of plastination, in which biologic tissues are impregnated by curable polymers [1]. This results in dry, odorless, and durable specimens that have intact gross and microscopic anatomy. However, the joint space itself and its recesses are not easily recognized because in the process of plastination the joint fluid is removed. Therefore we decided to combine plastination with arthrography. In this way, both the joint space and its outlining structures can be depicted.

In this article we describe our experiences with two techniques of plastination arthrography. The images obtained by plastination arthrography were correlated with images of MR arthrography and were used to study the normal anatomy of the distal tibiofibular syndesmosis.

\section{Materials and methods}

We used six fresh frozen human cadaveric lower legs, which were amputated through the knee joint. The specimens were thawed $12-24 \mathrm{~h}$ before scanning. In all specimens both a conventional MR as well as MR arthrography 
were performed. As contrast agent we used Gadolinium (Magnevist $^{\circledR}$ dimeglumingadopentetate, Schering, Germany; Gd) in MR and dyed polymer in plastination. After MR imaging the leg was frozen again for a week at $-20^{\circ} \mathrm{C}$ in order for the intra-articular polymer to cure. After this, $3-\mathrm{mm}$ slices of fresh frozen legs were obtained in a $45^{\circ}$ oblique axial plane identical to that of the MR images. These slices were then plastinated according to the E12 technique of von Hagens. The plastination slices were subsequently compared with MR images.

The injection of contrast fluid for MR-plastination arthrography was performed in either a one-step or a twostep procedure. In the one-step procedure MR arthrography was performed after a single injection with a mixture of $\mathrm{NaCl}(0.9 \%), \mathrm{Gd}$, and dyed polymer. In the two-step procedure injection of a mixture of $\mathrm{NaCl}(0.9 \%)$ and $\mathrm{Gd}$ for MR arthrography was followed by removal of the Gd and a second injection of dyed polymer for plastination arthrography. To prevent artifacts at the level of the anterior syndesmosis, injection was performed between the tendon of the anterior tibial muscle and the medial malleolus at the level of the talocrural joint.

For the one-step procedure it was necessary to find the optimal concentration of $\mathrm{Gd}$ and the optimal curation speed of the polymer solution. Two series with each nine different Gd concentrations were made in test tubes. The dilutions of Gd in these tubes increased from 1:50-1:250 with steps of 25 , with $\mathrm{NaCl}(0.9 \%)$ as the diluting agent. The first series was made with a fast-curing polymer, the second series with a slow-curing polymer. The slow-curing polymer was made with half the amount of hardening component (Biodur Härter E2) of that used in the fast-curing polymer.
The 18 test tubes were scanned axially using T1weighted FFE images (TR $\left.=5.1 \mathrm{~ms}, \mathrm{TE}=1.8 \mathrm{~ms}, \alpha=55^{\circ}\right)$, a $128 \times 256$ matrix, NSA $=1$, and one slice with a thickness of $10 \mathrm{~mm}$ at a $1.5 \mathrm{~T}$ MRI (Gyroscan, Philips, Best, The Netherlands). The tubes were scanned at 2-min intervals for a period of $8.5 \mathrm{~h}$. The change in signal intensity with time was plotted in time-intensity curves. The time available to perform MR-plastination-arthrography was determined by the time the signal of the mixture had faded for $50 \%$.

A 1.5 T MR (Gyroscan, Philips, Best, The Netherlands) and $\mathrm{C} 3$ surface coil around the cadaver ankles were used to obtain high-resolution images of the distal tibiofibular syndesmosis. The syndesmosis was scanned in a coronal, an axial, and an oblique axial plane. The latter plane had shown to give the best images of the syndesmosis in a previous study $[2,3]$. T1-weighted images were acquired with a matrix of $512 \times 512$, a field of view of $120-140 \mathrm{~mm}$ and 2.5-mm slice thickness. For the MR arthrography T1weighted images with spectral fat suppression (SPIR) were obtained using a matrix of $512 \times 512$, a field of view of 120-140 $\mathrm{mm}$ and 3.0-mm slice thickness. With these scan parameters an in plane resolution of about $250 \times 250 \mu \mathrm{m}$ was realized. Cod oil markers were fixed to the leg before scanning started in order to be able to define the exact plane in which the ankle had to be sawed [4].

\section{Results}

With time the signal intensity of the mixtures of Gd and polymer decreased as is demonstrated by the time-intensity curves (Fig. 1). The time-intensity curves showed the
Fig. 1 Signal intensity (SI (\%)) versus scan time (min) of two series of mixtures with polymer and Gadolinium (Gd) with different curing speed. $I$ fast curing, II slow curing polymer. Each series contains nine different $\mathrm{Gd}$ dilutions varying from $1: 50$ to $1: 250$, in steps of 25 $(1-9)$

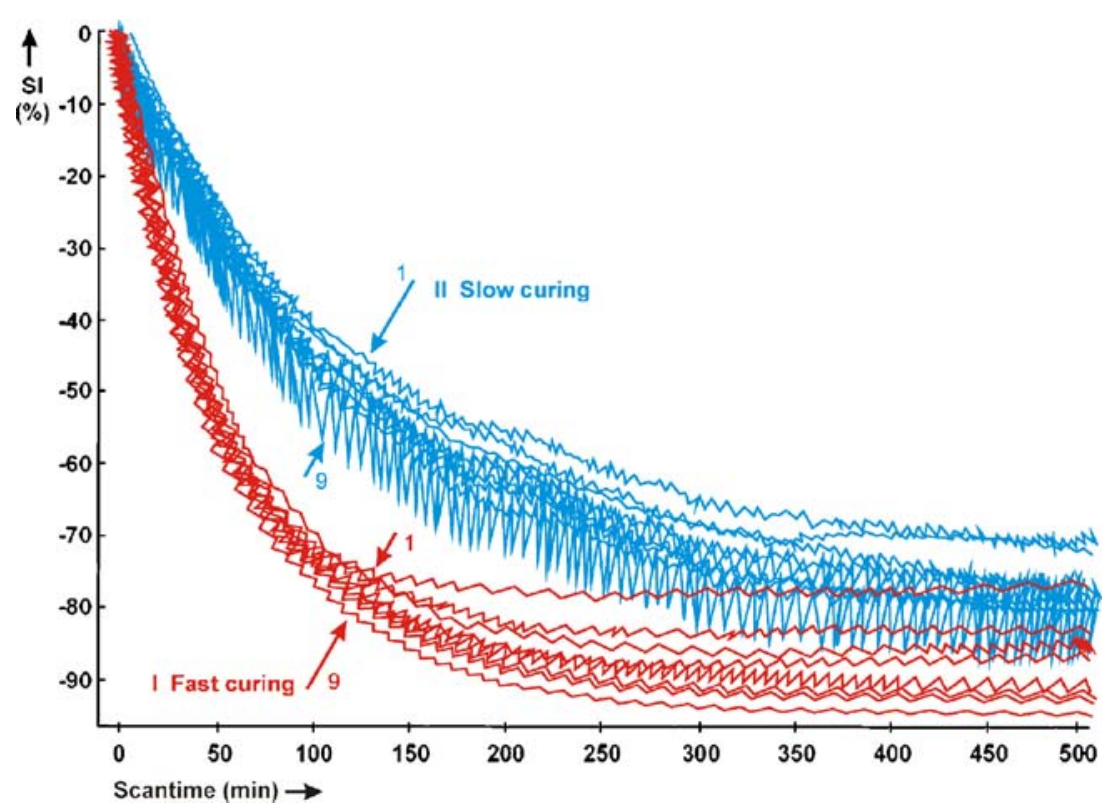




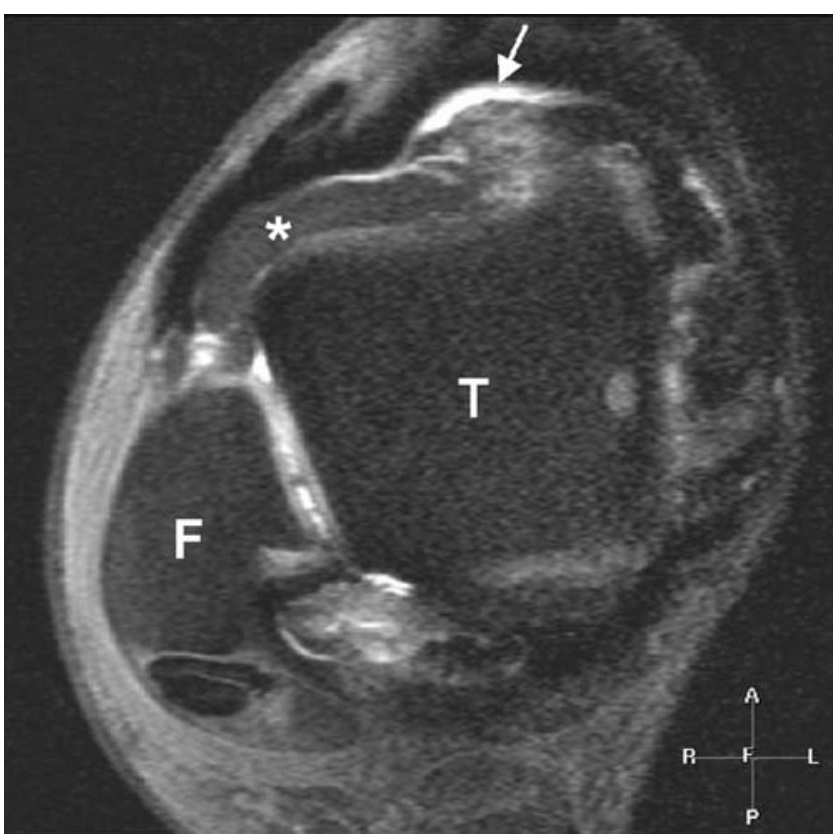

Fig. 2 Axial T1-w image with fat suppression after an intra-articular injection with a mixture of diluted $\mathrm{Gd}$ and polymer (one step procedure). $F$ fibula, $T$ talus. The arthrogram is inhomogeneous: there are both areas with high signal intensity (arrow) as well as areas with signal voids (asterisk) following: the decrease in signal intensity was initially greater in the fast-curing polymer than in the slow-curing polymer; after $512 \mathrm{~min}$ both polymers still had not completely solidified as the signal intensity of both the fast- and slow-curing polymer had not reached zero yet; at higher dilutions the decrease in signal intensity occurred at a greater rate than at lower dilutions.

MR-plastination-arthrography was performed in either a one- or a two-step procedure. For the one-step procedure we used the mixture with the slow-curing polymer with 1:50 diluted Gd. Although this procedure resulted in a good plastination arthrogram, the MR arthrogram appeared to be inhomogeneous (Fig. 2).

For the two-step MR plastination arthrography, we first performed a conventional MR arthrogram with 1:250 diluted $\mathrm{Gd}$, followed by an intra-articular injection of dyed polymer. This resulted in both a homogeneous MR arthrogram as well as a good plastination arthrogram.

The images acquired after the two-step MR plastination arthrography were compared with the plastination arthrography and showed an excellent correlation (Fig. 3). The talocrural joint space and its recesses were filled with contrast agent in the MR arthrogram and with dyed polymer in the plastination arthrogram. The syndesmotic
Fig. 3 Correlation between noncontrast enhanced MRI (a), plastination arthrography (b), and MR arthrography $(\mathbf{c}, \mathbf{d})$ at the level of the syndesmosis. The green dye (b) and contrast $(\mathbf{c}, \mathbf{d})$ are visible in the syndesmotic recess. The recess is not visible in the noncontrast MRI (a). The anterior and posterior tibiofibular ligaments outline the syndesmotic recess. The fat pad is visible behind the anterior tibiofibular ligaments $(\mathbf{a}, \mathbf{b})$. The cod oil markers are visible as white balls on the skin (a, c)
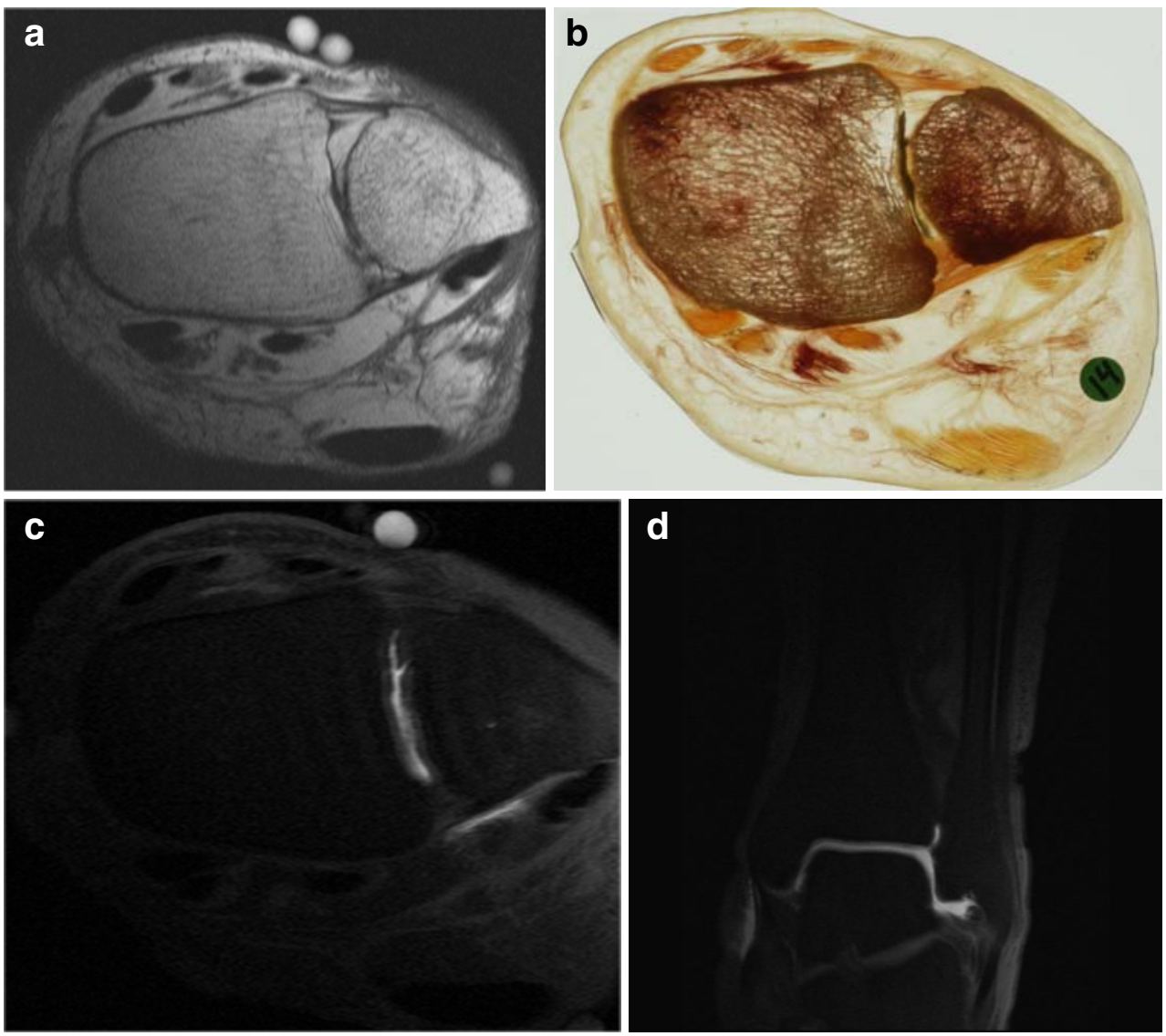


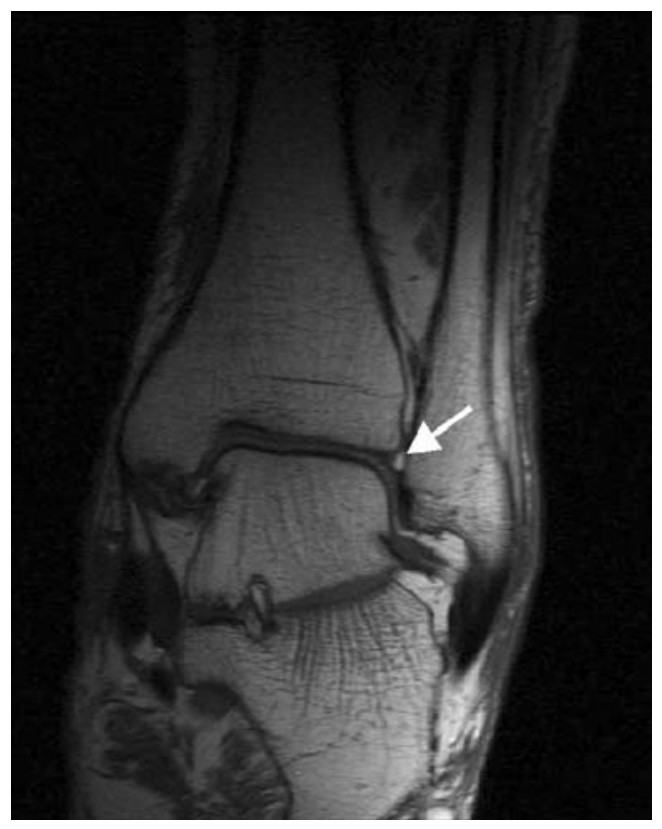

Fig. 4 Coronal T1-w image. A small fat pad (arrow) protrudes into the tibiotalar joint

recess was clearly visible in both the MR and the plastination arthrogram and the inner boundaries of the joint could easily be identified. The noncontrast enhanced MR images demonstrated ligaments, muscles, tendons, and the neurovascular bundles in great detail. The same structures were clearly recognizable in the plastinated slices.

Directly behind the anterior tibiofibular ligament was a small fat pad, which protruded variably into the talocrural joint (Fig. 4). In the T1-weighted images, the fat pad had the same signal intensity as the subcutaneous fat. In the MR arthrogram, a technique with fat suppression was used. The fat could now be seen as a signal void abutting the posterior border of the anterior tibiofibular ligament and indenting the syndesmotic recess (Fig. 3).

The anterior and posterior tibiofibular ligaments were only partially depicted in the axial images of the talocrural joint. However, in the oblique plane, running $45^{\circ}$ in caudalcranial direction from the lateral malleolus and related to the tibia plafond, the syndesmotic ligaments were depicted in their entire length (Fig. 5).

The transverse ligament was best seen in an axial image since it runs parallel to the tibial plafond between the medial and lateral malleolus and forms a labrum-like structure at the posterior distal tibia. We cannot present a plastination image of this ligament in the axial plane since we only made images in the aforementioned oblique plane. The interosseous membrane was well visualized in both the axial and the oblique plane since it constitutes a continuous sheet between the crest of the tibia and fibula.

\section{Discussion}

Several techniques can be used to perform MR arthrography. All techniques however are based on the presence of an intra-articular contrast-enhancing agent and on distension of the joint. A joint effusion or hemarthrosis, due to an acute or chronic injury of the joint, leads to distension of the joint with the intra-articular fluid acting as a natural contrast agent. Another way to perform an arthrogram is by injecting a saline solution into the joint and injecting $\mathrm{Gd}$ intravenously. The most commonly used technique however, is an intra-articular injection of a diluted Gd solution [5]. This results in a high intra-articular signal and therefore a good depiction of the intra-articular anatomy.

Until now no technique was available to perform plastination arthrography. Plastination is a process in which tissues are dehydrated and impregnated with a polymer. It is an excellent method to study anatomy, but as fluids are taken out of the tissues during the process, it is difficult to study intra-articular anatomy. Plastination arthrography could be the solution for this problem. When a polymer is added to the Gd solution there will be a decrease in signal intensity with time because curing of the polymer results in
Fig. 5 Axial (left) and $45^{\circ}$ oblique (right) T1-w image. In the axial image the anterior tibiofibular ligament looks interrupted (arrow). In the oblique image the ligament is depicted along its entire length (arrow)
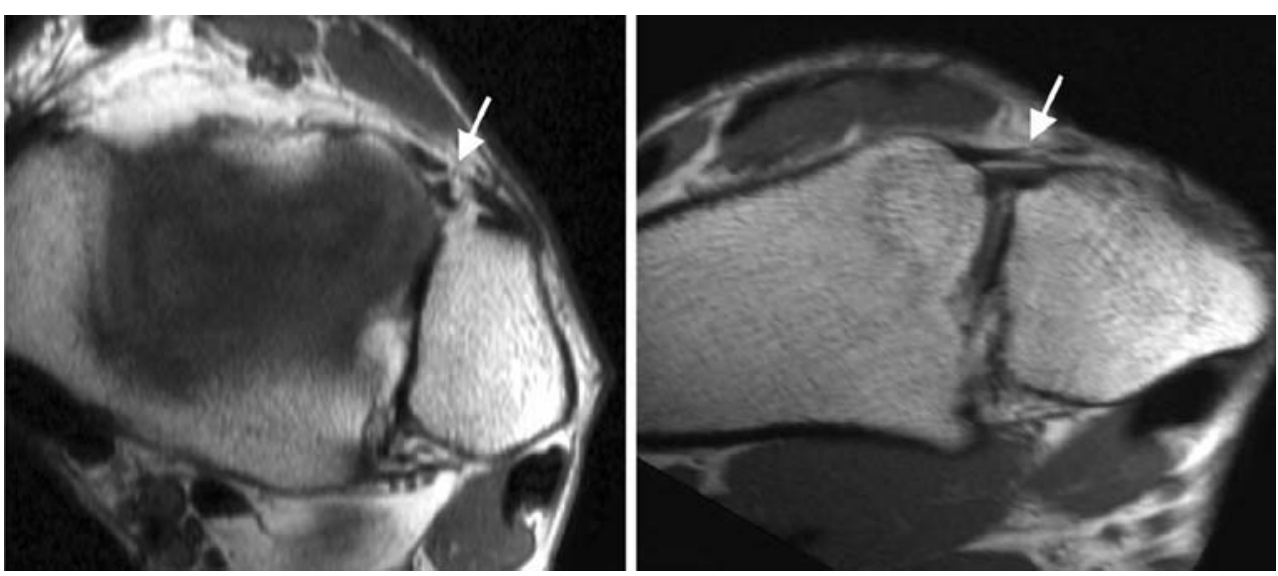
an increasing solidity of the mixture. This decrease in signal intensity goes on until, finally, there is a signal void.

In order to be able to perform MR arthrography, we looked for that specific mixture of $\mathrm{Gd}$ and polymer, which would give us the maximum time window to scan before the signal intensity, dropped below a visible level. The mixture showing the highest signal intensity for the longest period of time was the slow-curing polymer with a 1:50 diluted Gd solution. At $50 \%$ decrease of the initial signal intensity, this Gd-polymer mixture was still clearly visible. The $50 \%$ reduction in signal intensity was reached after $150 \mathrm{~min}$. Since we needed circa $50 \mathrm{~min}$ to prepare the $\mathrm{Gd}-$ polymer mixtures before we could start MRI scanning, the total available time window before the signal intensity dropped below a visible level added up to $200 \mathrm{~min}$. This gave us ample time to perform the MR imaging.

The most convenient way to perform MR plastination arthrography is with a single intra-articular injection of a mixture of Gd and polymer. Such a mixture did not give a good result in this study. Although the joint could be clearly visualized, the arthrogram was inhomogeneous. As such it was not possible to study the joint lining and syndesmotic recess. There could be a number of reasons for this phenomenon.

First, the ongoing process of curing could be the cause of this inhomogeneous arthrogram. However, this is not likely since all images were acquired within approximately 110 min after we started the preparation of the Gd-polymer while the total available time window amounted $200 \mathrm{~min}$.

Secondly the presence of joint fluid and/or the presence of tissues forming the joint lining may give an additional interaction between the Gd and polymer. This could lead to a faster decrease of signal intensity in the joint than was observed in the test tubes. Moreover the curing process itself may be an inhomogeneous process.

Changing the order of mixing the components of the polymer solution with the hardening component (Biodur Härter E2) added last however, did not result in a more homogenous arthrogram. We therefore changed to a twostep procedure with a conventional MR arthrogram as a first step, followed by an intra-articular injection of a dyed polymer and plastination of the leg.

The obliquely running anterior and posterior tibiofibular ligaments are only partially visible in axial images of the talocrural joint. This may lead to the erroneous interpretation of a rupture of these ligaments. Generally, a ligament is best depicted in a plane along its length. We therefore scanned and sawed the specimen in an oblique plane, which was defined with the aid of markers containing cod oil, which were attached to the skin of the cadaveric leg.

This article shows that the study of clinically relevant anatomical structures clearly improves by using plastination arthrography. This can best be done following the two-step procedure. The first step was the MR arthrogram with a 1:250 diluted Gd solution; the second step was the plastination arthrogram with a dyed polymer. With this technique we achieved an excellent correlation between MR images and plastinated slices of the distal tibiofibular syndesmosis. MR-plastination-arthrography demonstrated the clinically relevant syndesmotic recess, the fat pad, and the tibiofibular ligaments in great detail. This technique can be applied to study the anatomy of any synovial joint. However, it is essential to obtain plastination slices in the same plane as the imaging slices. To optimize this technique for each joint may be a subject for future studies.

Open Access This article is distributed under the terms of the Creative Commons Attribution Noncommercial License which permits any noncommercial use, distribution, and reproduction in any medium, provided the original author(s) and source are credited.

\section{References}

1. Von Hagens G, Tiedemann K, Kriz W. The current potential of plastination. Anat Embryol 1987; 175: 411-421.

2. Hermans JJ, Beumer A. MRI Characteristics of chronic syndesmotic injuries. ISMRM, Tenth Scientific Meeting and Exhibition, 18-24 May 2002 Honolulu, Hawaii, USA.

3. Beumer A, Hermans JJ, Niesten DD, Heijboer RP. Late reconstruction of the anterior tibiofibular syndesmosis for ankle diastasis with talar shift in a 12-year-old boy. A case report. Foot Ankle Surg 2005; 11: 49-53.

4. Entius CAC, Kuiper JW, Koops W, de Gast A. A new positioning technique for comparing sectional anatomy of the shoulder with sectional diagnostic modalities: magnetic resonance imaging (MRI), computed tomography (CT) and ultrasound (US). J Int Soc Plastination 1993; 7: 23-26.

5. Lee SH, Jacobson J, Trudell D, Resnick D. Ligaments of the ankle: normal anatomy with MR arthrography. J Comput Assist Tomography $1995 ; 22(5)$ : 807-813. 